\section{A Microwave Direction of Arrival Estimation Technique Using a Single Antenna}

Xiaoju Yu, Rongguo Zhou, Hualiang Zhang, and Hao Xin

\begin{abstract}
A direction of arrival (DoA) estimation technique for broadband microwave signals is proposed using a single Ultra-Wide-Band (UWB) antenna. It is inspired by the sound source localization ability of human auditory system using just one ear (monaural localization). By exploiting the incident angle dependent frequency response of a wide-band antenna, the DoA of a broadband microwave signal can be estimated. DoA estimation accuracies are evaluated for two antenna configurations and microwave signals with different signal-to-noise ratios (SNR). Encouraging DoA estimation performance of the proposed technique is demonstrated in both simulation and experiment.
\end{abstract}

Index Terms - Biological Inspired, Direction of Arrival (DoA) Estimation, Incident Angle Dependent Spectra, UWB Antenna

\section{INTRODUCTION}

IN recent years, there has been increasing interest in microwave direction finding systems due to their wide applications in military and commercial areas, such as electronic warfare [1], wireless communications [2], wireless localization, etc. A wide variety of techniques have been developed to estimate the direction of arrival (DoA) of incoming signals, including the estimation of signal parameters via rotational invariance techniques (ESPRIT) [3] and multiple signal classification (MUSIC) [4], etc. All of these techniques require a large number of antenna elements to achieve a high degree of accuracy. However, as the number of antenna elements increases, the power consumption, size and cost of the system increase as well. Therefore, accurate DoA estimation technique with reduced number of antenna elements is highly desirable.

To achieve compact microwave direction finding systems, several biomimetic techniques have been proposed. Inspired by the human auditory system, an improved DoA estimation technique using just two antennas with a scatterer in between them to emulate the function of human head as a low pass filter, has been reported [5, 6]. An array with two electrically small antennas with enhanced directional sensitivity is demonstrated in [7] mimicking parasitoid fly. It turns out that another amazing capability of the human auditory system is the monaural direction finding. Although not as accurate as the binaural (utilizing both ears) case, monaural direction finding is possible without head movement. This first seems to be

Manuscript received April 11 $1^{\text {th }}, 2016$.

$\mathrm{X}$. Yu and $\mathrm{H}$. Xin are with the Department of Electrical and Computer Engineering, University of Arizona, Tucson, AZ 85721, USA (e-mail: hxin@ece.arizona.edu).

R. Zhou and H. Zhang were with the Department of Electrical and Computer Engineering, University of Arizona, Tucson, AZ 85721, USA. R. Zhou is now with Infineon Technologies, Morgan Hill, CA 95037, USA. H. Zhang is now with the Department of Electrical and Computer Engineering, University of Massachusetts Lowell, Lowell, MA 01854, USA. intuitively unthinkable for a single stationary antenna. However, the monaural localization only works for broadband signals. The main mechanism has been identified to be the spectral alteration by the pinnae and head that provides cue for directions (similar to an incident angle sensitive "comb-line filter") [8, 9]. For example, as a simplified illustration shown in Fig. 1, the received broadband signal by a single ear may have a notch response that is incident angle dependent. Therefore, monaural direction finding works only for broadband signals. Experimentally measured frequency-dependent single ear head-related transfer functions (HRTF) for both azimuth and elevation planes had been reported in [10]. By designing the frequency dependences of antenna pattern and guiding structure, this unique feature of human ear may be utilized for microwave applications such as direction finding for an ultra-wide-band (UWB) signal [11].

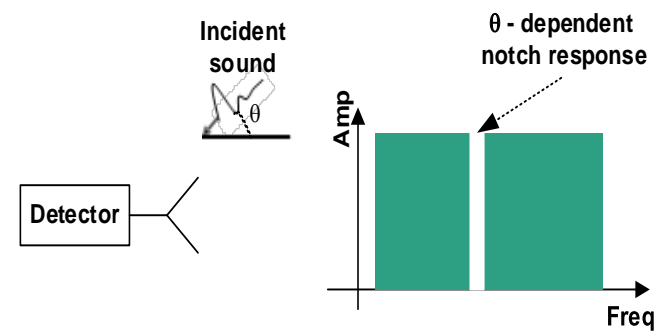

Fig. 1. Simplified illustration of monaural direction finding of human auditory system: the received amplitude spectrum of an incoming broadband signal depends on the incident angle of the signal.

In [12], a previously demonstrated linear-polarized elliptical UWB (3.1 to $10.6 \mathrm{GHz}$ ) antenna [13] was used to investigate the single antenna direction finding technique. The UWB antenna enables DoA estimation for a broadband signal using incident angle dependent power spectra. The DoA estimation accuracy of this technique was theoretically studied in all three planes with the assumption of different signal to noise ratios (SNRs). In this paper, first, the DoA estimation performance is re-evaluated in more realistic scenarios including a constant noise level, transmission spectral distortion and multipath effect. Moreover, an improved UWB antenna with asymmetrical shape (D shape) is designed and studied, demonstrating improved estimation accuracy due to the break of symmetry. Finally, Both UWB antennas are fabricated and their DoA estimation performance is tested using a horn antenna as the illuminator. The measured results confirm the feasibility of the proposed single antenna DoA estimation technique. This paper is organized as the following: Problem setup and simulation results of the DoA estimation performance are presented in Section II. Section III presents the experimental verification of this single-antenna DoA estimation method. Finally, brief conclusions and discussions are given in Section IV.

\section{II.EXPERIMENTAL SETUP AND SimUlATED PERFORMANCE ANALYSIS}

A single UWB antenna is used to estimate the DoA of incident broadband microwave signals. Similar to human monaural sound localization, the received spectra of the UWB antenna are correlated to the DoA. Therefore, the DoA of a 
broadband signal received by the antenna can be estimated by evaluating the correlation coefficients of the measured spectrum with pre-determined incident angle dependent spectra. To implement this idea in microwave DoA estimation applications, two UWB antennas, one previously demonstrated with elliptical configuration [12] and another modified antenna with D-shaped configuration for improved performance are investigated. The experimental setup is presented first. The corresponding simulated DoA estimation performance using the two UWB antennas is presented next.

\section{A. Experimental Setup}

Experimental setup of the single antenna direction finding system is shown in Figs. 2 and 3. Measurements are performed on the roof of a building. The UWB receiver under test is placed in the far field zone of the transmitter. The transmitted UWB signal $(3-10 \mathrm{GHz})$ in the experiment is realized by continuous wave (CW) frequency sweeping. Figure 3 illustrates the UWB antenna orientations under test mounted for the measurement of incident waves in the $x-y$ plane, the $x-z$ plane, and the $y-z$ plane. The coordination is as shown in Figs. 4 and 6.

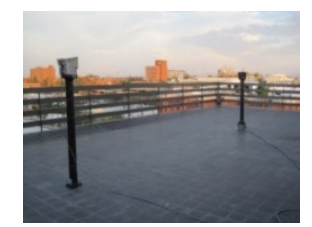

Fig.2. Measurement setup with transmitter and receiver on a building roof.
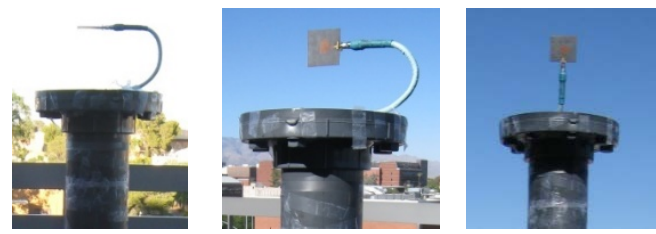

Fig.3. UWB Receiver set up for incident-angle sweeping in the x-y plane (left), the $\mathrm{x}-\mathrm{z}$ plane (middle), and the $\mathrm{y}-\mathrm{z}$ plane (right).

\section{B. Elliptical UWB Antenna and DoA Estimation}

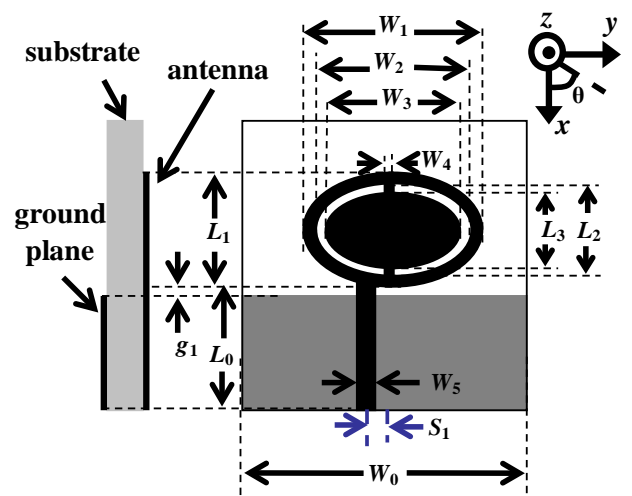

Fig. 4. The schematics (left: side view; right: top view) of the elliptical UWB antenna with two symmetric slots [12].

The antenna response is modeled by the full-wave finite-element electromagnetic solver - High Frequency Structure Simulator (HFSS) [14]. The schematic of the elliptical antenna is shown in Fig. 4 and the details can be found in [12]. This antenna is linearly polarized with its co-polarization and cross-polarization similar to those of a monopole. When the incident wave is cross polarized, the power level received at the antenna is much lower than that of the co-polarizations, resulting in a poor performance of incident angle estimation. For this reason, only system performances with incident waves of co-polarization are analyzed.

To take into account noises, Gaussian White Noise (GWN) is added to the received spectra $(3-10 \mathrm{GHz})$. The SNR used in the simulation is the maximal received SNR, considering the main-beam direction of the antenna. Therefore, the actual SNR has angular dependence due to the radiation pattern of the antenna. The cross-correlation coefficients between the spectra amplitude with added noises (emulating measured signal) and the original spectra amplitude are then calculated for all incident angles. The DoA of the signals with noise is then estimated by selecting the angle with the largest correlation coefficient.

(a)

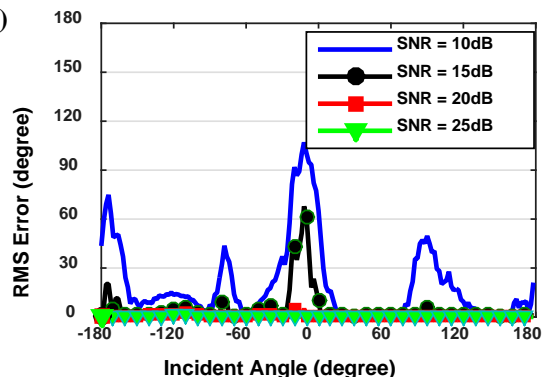

(b)

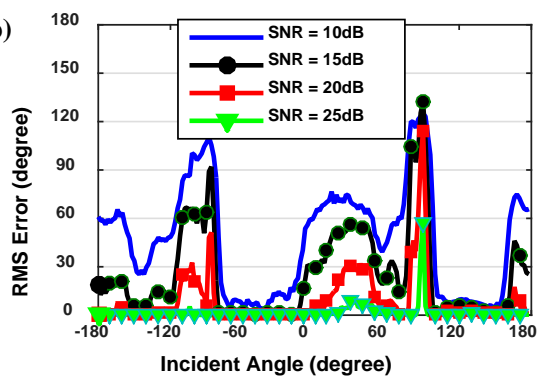

(c)

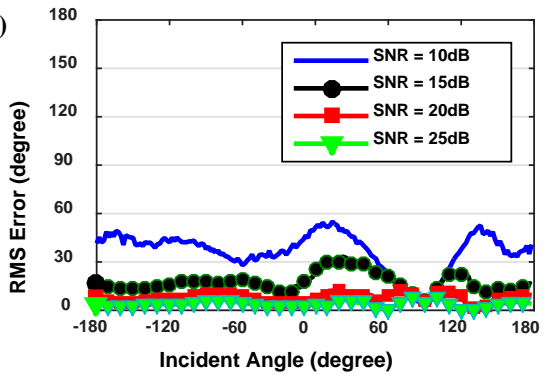

Fig. 5. The RMS of the estimation error with different SNRs for incident waves in (a) the $x$-y plane (H-field in the $z$ direction), (b) the $x$-z plane (H-field in the $y$ direction), and (c) the $y$-z plane (E-field in the $x$ direction).

Figure 5(a) plots the Root Mean Square (RMS) of the estimation errors against incident angles in the $x-y$ plane with $\mathrm{H}$-field in the $z$ direction for different SNRs. The RMS errors are calculated by running the simulation 1000 times. The estimation errors are less than $6^{\circ}$ with most incident angles for $\mathrm{SNR} \geq 15 \mathrm{~dB}$ cases. With the decrease of SNR, the RMS of the estimation error increases due to the influence of the noise. This is quite similar to the human monaural sound localization performance [8]. The high estimation errors around $0^{\circ}$ and $180^{\circ}$ when SNR $\leq 15 \mathrm{~dB}$ are due to relatively low received power levels at these angles ( $20 \mathrm{~dB}$ lower than the maximum possible received power of the antenna). Similarly, the received spectra of the UWB antenna in the $x$-z plane with H-field in the $y$ 
direction, and in the $y$-z plane with E-field in the $x$ direction are also direction dependent, to different degrees [12]. For co-polarization in the $\mathrm{x}-\mathrm{z}$ plane (Fig. 5(b)), the estimation errors are less than $10^{\circ}$ for most angles with $S N R \geq 20 \mathrm{~dB}$. When SNR $<20 \mathrm{~dB}$, large estimation errors occur. Large peaks of error around $+/-90^{\circ}$ are due to the fact that the received power levels are low at those angles (same as spectra around $0^{\circ}$ and $180^{\circ}$ in the $x-y$ plane). Large error peaks around $0^{\circ}$ and $180^{\circ}$ are due to the relatively small angular dependence of the spectra (structure symmetry). For incident waves in the $y$-z plane with E-field in the $\mathrm{x}$ direction, the received power levels are large for all the angles compared with other polarizations. The RMS estimation errors are smaller than $30^{\circ}$ when $\mathrm{SNR} \geq 15 \mathrm{~dB}$. There is almost no region for low estimation errors when SNR is low $(\mathrm{SNR}=10 \mathrm{~dB})$ because of the relatively small angular dependence of the spectra [12].

\section{C.D-Shaped UWB Antenna and DoA Estimation}

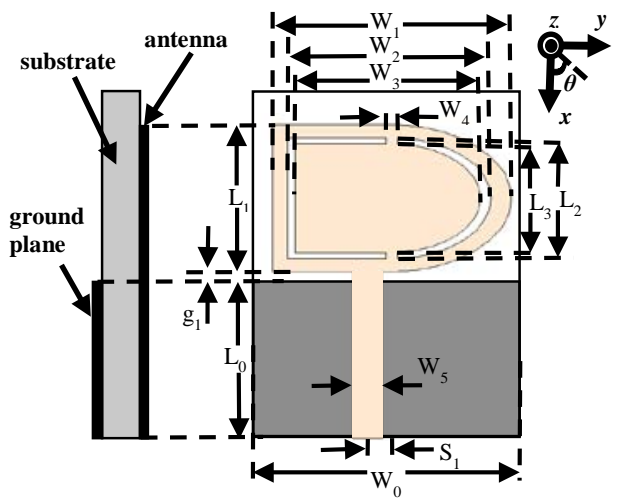

Fig. 6. The schematics (left: side view; right: top view) of a D-shaped UWB antenna.

Due to its symmetry with respect to the $x$-axis, the spectra of the elliptical UWB antenna with added noise are also correlated to the original spectra, along the symmetric direction with respect to the $x$-axis plane (i.e., $\theta$ and $-\theta$ ). To break this symmetry to increase the estimation accuracy, another UWB antenna with a D-shaped configuration is also designed, as shown in Fig. 6. The antenna design parameters are selected to be: $L_{0}=16 \mathrm{~mm}, L_{1}=14 \mathrm{~mm}, L_{2}=11.6 \mathrm{~mm}, L_{3}=10.4 \mathrm{~mm}, S_{1}=$ $2 \mathrm{~mm}, W_{0}=23 \mathrm{~mm}, W_{1}=19.6 \mathrm{~mm}, W_{2}=16.72 \mathrm{~mm}, W_{3}=15.28$ $\mathrm{mm}, W_{4}=1 \mathrm{~mm}, W_{5}=2.4 \mathrm{~mm}$ and $g_{1}=0.3 \mathrm{~mm}$. The simulated reflection coefficient of this antenna is less than $-6 \mathrm{~dB}$ from 3 to $10 \mathrm{GHz}$, as shown in Fig. 12(b).

Following the same DoA estimation procedure described previously, the correlation coefficients of the spectra with and without added noise (SNR $=15 \mathrm{~dB}$ ) are plotted in Fig. 7(a), for the case of incident waves in the $x$-y plane ( $\mathrm{H}$-field in the $z$ direction). The corresponding RMS of the estimation errors with different SNRs are plotted in Fig. 7(b). The spectra with added noises are still highly correlated to the original spectra along the direction of incident signal, as expected. In addition, the correlation coefficients at the symmetric direction with respect to the $x$-axis are reduced due to the break of symmetry of the D-shaped antenna, leading to improved estimation accuracy. The RMS of the estimation error is less than $6^{\circ}$ for $\mathrm{SNR} \geq 15 \mathrm{~dB}$ and better than the case of elliptical antenna (when $\mathrm{SNR}=15 \mathrm{~dB}$, averaged RMS error for D-shaped antenna case is $2^{\circ}$ while elliptical antenna case is $5^{\circ}$ ), as shown in Fig. 7 (b). The higher RMS errors around $0^{\circ}$ and $180^{\circ}$ are due to lower received power level.

(a)

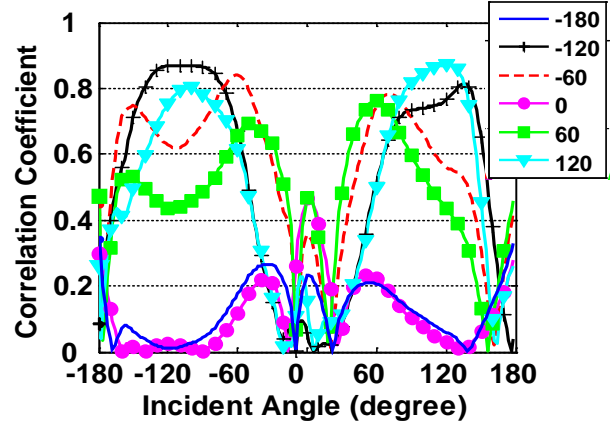

(b)

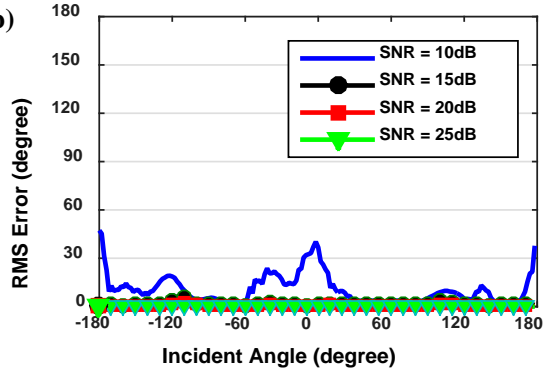

Fig. 7. DoA estimation performance of the D-shaped UWB antenna in the $x-y$ plane (H-field in the $z$ direction): (a) The correlation coefficients between spectra with added noise (SNR $=15 \mathrm{~dB}$ ) and the pre-determined spectra at the incident angle $\theta\left(-180^{\circ},-120^{\circ},-60^{\circ}, 0^{\circ}, 60^{\circ}\right.$ and $\left.120^{\circ}\right)$; (b) The RMS of the estimation errors for different SNRs.

(a)

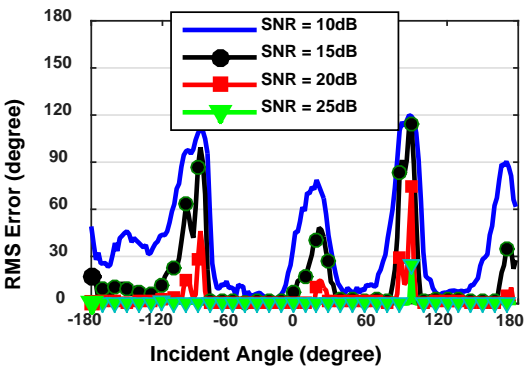

(b)

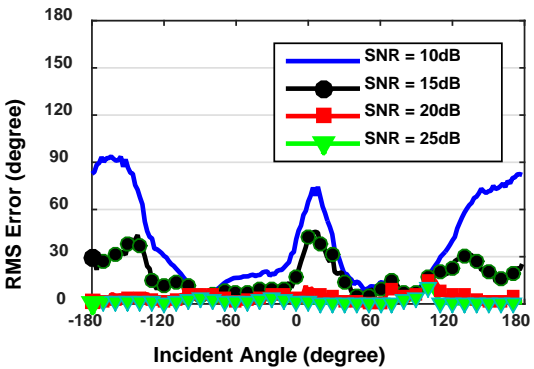

Fig. 8. The RMS of the DoA estimation errors of the D-shaped antenna for different SNRs when incident waves are in (a) the $x$ - $z$ plane (H-field in the $y$ direction), and (b) the $y-z$ plane (E-field in the $x$ direction).

Figure 8 (a) and (b) plot the RMS errors when the incident waves are in the $\mathrm{x}-\mathrm{z}$ plane (H-field in the $y$ direction) and $y-z$ plane (E-field in the $x$ direction) respectively. For the case of incident waves in the $x-z$ plane with co-polarization, the RMS errors have peaks around $0^{\circ}, 180^{\circ}$ and $+/-90^{\circ}$ because of the same reason as the elliptical antenna case. Due to the break of symmetry respect to $\mathrm{x}$ axis, the RMS errors in the $y$-z plane is lower compared to the elliptical antenna case for most incident angles except for around $0^{\circ}$ and $180^{\circ}$ where symmetry respect to y axis still exists. 

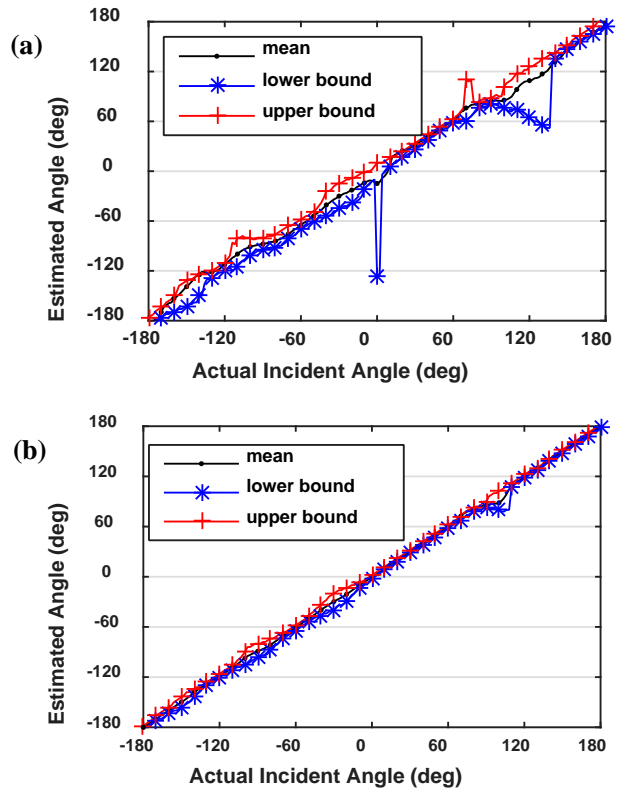

Fig. 9. Estimated DoA (mean values, lower and upper bound at $90 \%$ confidence level) versus true incident angles at SNR levels of $15 \mathrm{~dB}$ (a) and $20 \mathrm{~dB}$ (b), in the $\mathrm{y}$-z plane (E-field in the $\mathrm{x}$-direction), for the D-shaped antenna.

For better understanding of the DoA estimation performance, Fig. 9 plots the estimated incident angles (mean, upper and lower bounds for $90 \%$ confidence level) versus the true incident angles for the D-shaped antenna. SNR levels of 15 and $20 \mathrm{~dB}$ in the $\mathrm{y}-\mathrm{z}$ plane (E-field in the $\mathrm{x}$-direction) are investigated. Figure 9(a) shows that when SNR is $15 \mathrm{~dB}$, the width of the confidence interval corresponds well with the RMS error plotted in Fig. 8(b). The larger the RMS value, the wider the calculated confidence interval is. When the confidence interval is as wide as $30^{\circ}$, the estimator has a $10^{\circ}$ bias (difference between average estimated angle and the true incident angle); when the confidence interval is narrow, a good estimation with no bias is obtained. As shown in Fig. 9(b), when the SNR $=20$ $\mathrm{dB}$, a good angle estimation with no bias and small deviation is obtained except around $100^{\circ}$. As analyzed, a high SNR is critical in the angle estimation. If the SNR was very low, the angle estimation would have not only large deviation but also biased average value. However, if the background noise is simply Gaussian White noise, averaging and smoothing can be used to increase the data SNR effectively.

\section{D.Impact of Spectral Distortion and Multipath Effect}

One caveat of this technique is that it does require the user to know the transmitted signal (including the transmitter's spectral response) a priori. Similarly, for the human auditory system, monaural DoA estimation works only when a white noise (or a signal close to white noise) is incident and / or after some prior self-training. To better understand this issue, the impact of spectral distortion of the transmitted signal is studied. The transmitted power spectral distortion is modeled as a function of frequency $\mathrm{f}$ as: $10^{-0.1 \alpha\left(\frac{2\left(\mathrm{f}-\mathrm{f}_{0}\right)}{\mathrm{BW}}\right)^{2}}$ or $-\alpha\left(\frac{2\left(\mathrm{f}-\mathrm{f}_{0}\right)}{\mathrm{BW}}\right)^{2} d B$, where $\alpha$ is the distortion level in $\mathrm{dB}, \mathrm{f}_{0}$ is the center frequency of the band, and BW is the bandwidth. The actual transmitted spectrum is the result of multiplying the original white spectrum by the power spectral distortion. For the case of the D-shaped UWB antenna (incident waves in the $x-y$ plane with
$\mathrm{H}$-field in the $\mathrm{z}$ direction), the average RMS estimation errors over all incident angles are simulated and plotted in Fig. 10. Various spectral distortion levels and SNRs assuming GWN are considered. As expected, higher spectral distortion level and lower SNR degrade the DoA estimation performance.

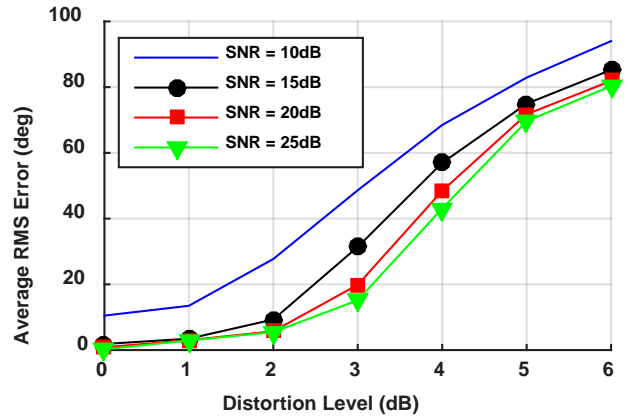

Fig. 10. Average RMS error of the DoA estimation over all incident angles vs. spectral distortion levels at various SNRs, for the D-shaped UWB antenna with incident waves in the $x-y$ plane (H-field in the $\mathrm{z}$ direction).

In addition, multipath effect is important for many practical applications. As an initial study, the impact of multipath effect on DoA estimation accuracy is investigated using a multi-ray statistical channel model [15]. When the number of rays / paths equals one, it means there is only the LOS propagation. All other cases include multipath fading channels in addition to the LOS path. Each multipath signal is assumed to have uniformly distributed additional path loss from $10 \mathrm{~dB}$ to $25 \mathrm{~dB}$ compared to the line-of-sight (LOS) path, uniformly distributed phase from 0 to $2 \pi$ at each frequency bin, and uniformly distributed arrival angle in the azimuth plane. For the case of the D-shaped UWB antenna (incident waves in the $x-y$ plane with $\mathrm{H}$-field in the $z$ direction), the DoA estimation performance vs. the number of propagation paths is plotted in Fig. 11. It can be observed that the resulting average RMS estimation error is larger with stronger multipath effect as expected. In general, the proposed DoA estimation method for CW based broadband signal is less robust when there is strong multipath effect. However, for applications in an open space - grassland, schoolyard, military base land, etc., the multipath effect is weak and less important. In addition, a pulse based UWB system may be used which is less prone to multipath fading compared to a CW based system. We have demonstrated such a pulse based system using a leaky wave antenna [16] with high accuracy in $90^{\circ}$ range.

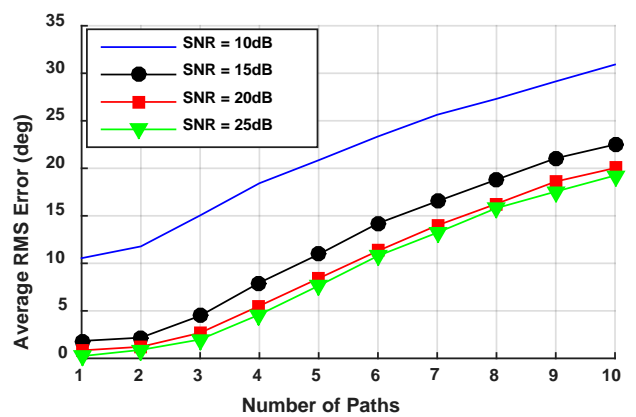

Fig. 11. Average RMS error of the DoA estimation over all incident angles vs. number of propagation paths at various SNRs, for the D-shaped UWB antenna with incident waves in the $\mathrm{x}-\mathrm{y}$ plane ( $\mathrm{H}$-field in the $\mathrm{z}$ direction). 


\section{EXPERIMENTAL VERIFICATION}

To verify the DoA estimation technique using a single UWB antenna experimentally, the two UWB antennas discussed in section II are fabricated and tested. The measured return losses of the elliptical and D-shaped antennas using Agilent E8361A vector network analyzer are plotted in Fig. 12. The measured reflection coefficients have some small frequency shifts compared to the simulation, which is likely due to the SMA connector effects and fabrication tolerances [13].
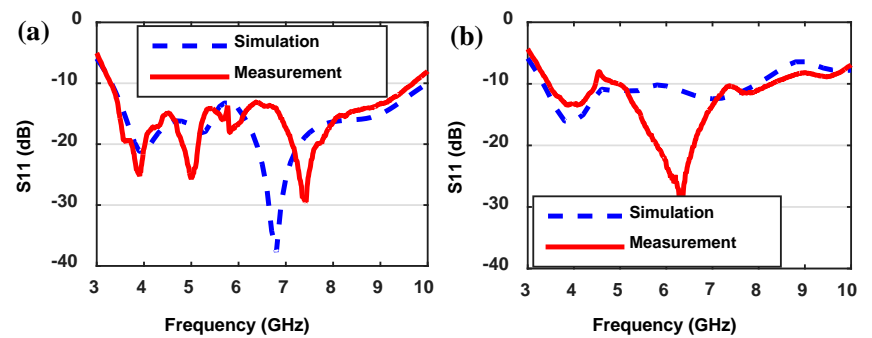

Fig. 12. Simulated and measured reflection coefficients of (a) the elliptical UWB antenna (b) the D-shaped UWB antenna.

The UWB receiver is placed $5.3 \mathrm{~m}$ away from the transmitter (in the far field zone of the transmitter) as shown in Fig. 2. The experiments are performed from 3 to $10 \mathrm{GHz}$ (with $0.01 \mathrm{GHz}$ step) using a vector network analyzer (Agilent E8361A). CW frequency sweeping is applied for the transmitted signal to obtain broadband spectra. However, in practice the system is applicable for broadband pulse signal with relatively flat frequency spectra. The source signal can have arbitrary frequency response if the transmitted waveform is known at the receiver a priori. The received spectra are measured for both UWB antennas in different planes for different polarizations. The transmitting antenna is a $1-18 \mathrm{GHz}$ ridged horn antenna. The source power is $+10 \mathrm{dBm}$. The UWB antennas are placed on the positioning system and rotated from $-180^{\circ}$ to $180^{\circ}$ with $2^{\circ}$ step horizontally.

Two sets of spectra as a function of incident angles are measured at different locations as the calibration data and testing data respectively. The estimated incident angle in testing is calculated by finding the angle with peak correlation coefficients between the tested spectrum and the pre-saved calibration spectra. When the incident wave is from the $\mathrm{z}$ direction and $\mathrm{E}$ field in the $\mathrm{x}$ direction, the received SNR is approximately $10-15 \mathrm{~dB}$. The measured spectra are distorted somewhat comparing to simulation which might be due to the following factors, first, the scattering of the feeding cable of the receiving antenna; second, the frequency dependent fading due to multipath effect of the environment; and last, the frequency response of the transmitting horn antenna. As shown in Fig. 3, the directly transmitted signals reaching the receiver are also incident onto the connected cables, potentially causing scattering and distorting the measured spectra. Because of the existence of the sidewalls and ground, there may be frequency dependent fading due to multipath effect. At last, the gain of the double-ridged horn antenna used in the experiment is frequency dependent throughout the tested band, thus has impact on the received spectra. Smoothing process, though cannot remove spectral distortion introduced above, can reduce uncorrelated noises like GWN. Smoothing is processed with $0.1-\mathrm{GHz}$ span in the test. (a)

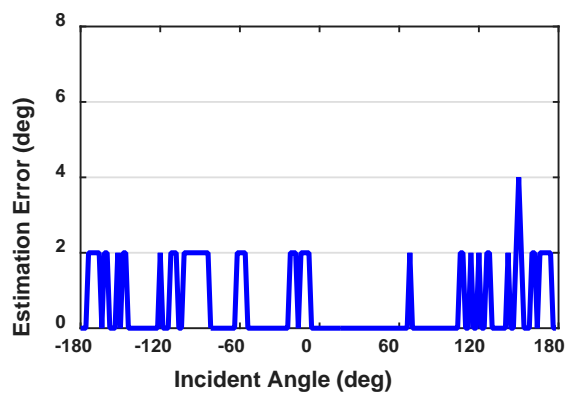

(b)

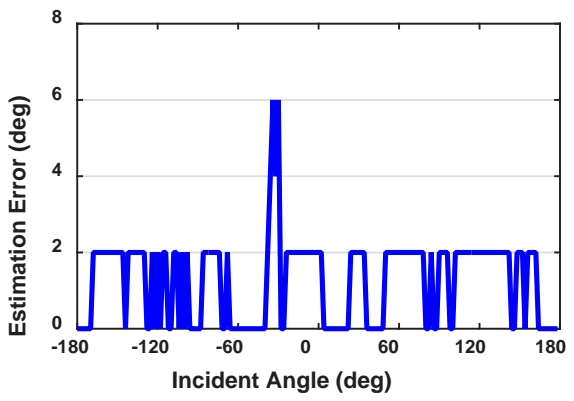

(c)

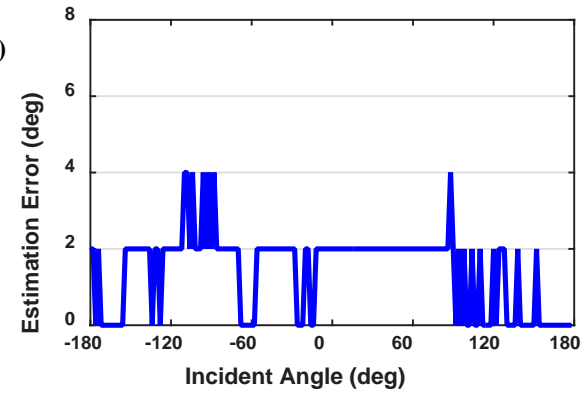

Fig. 13. The measured estimation errors of the elliptical UWB antenna with incident waves in (a) the $x-y$ plane (H-field in the $z$ direction), (b) the $x$-z plane (H-field in the $y$ direction), and (c) the $y$-z plane (E-field in the $x$ direction).

The estimation errors using the elliptical and the D-shaped UWB antenna are plotted in Figs. 13 and 14, respectively. For the elliptical antenna, as shown in Fig. 13, the measured estimation errors are less than $6^{\circ}$. The repeatability of this measurement is also verified by repeating the measurements in different days.

As shown in Fig. 14, the DoA estimation errors of the D-shaped antenna are less than $4^{\circ}$ for most incident angles and all cases. The large estimation errors at $0^{\circ}$ and $180^{\circ}$ in the $x-y$ plane with $\mathrm{H}$-field in the $\mathrm{z}$ direction (Fig. 14(a)), and at $+/-90^{\circ}$ in the $\mathrm{x}-\mathrm{z}$ plane with $\mathrm{H}$-field in the $\mathrm{y}$ direction (Fig. 14(b)), correspond with minimum received power at these angles. The elliptical antenna also has this issue. Simulation results successfully predict these peak estimation errors (see Figs. 7(b), 8(a)). Other than those angles, the D-shaped antenna has similar estimation performance compared to the elliptical antenna for $\mathrm{x}-\mathrm{y}$ and $\mathrm{x}-\mathrm{z}$ planes. Compared to the elliptical antenna, the D-shaped UWB antenna has slightly better angle estimation performance for the $\mathrm{y}$-z plane with E-field in the $\mathrm{x}$ direction due to symmetry broken as shown in Fig. 14(c). In general, the feasibility of reasonable DoA estimation using a single UWB antenna is verified.

At last, higher degree of estimation accuracy could be achieved using this single antenna DoA estimation technique with improved SNR, as discussed in Section II. We have demonstrated good $90^{\circ}$ range DoA estimation utilizing a 
microstrip leaky wave antenna, which has good spectra sensitivity and high SNR for all angles in the range [16].

(a)

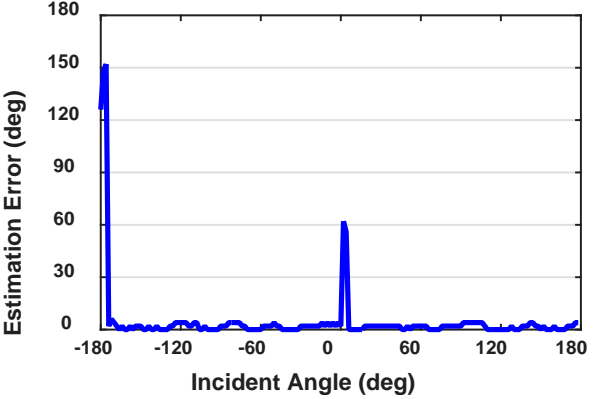

(b)

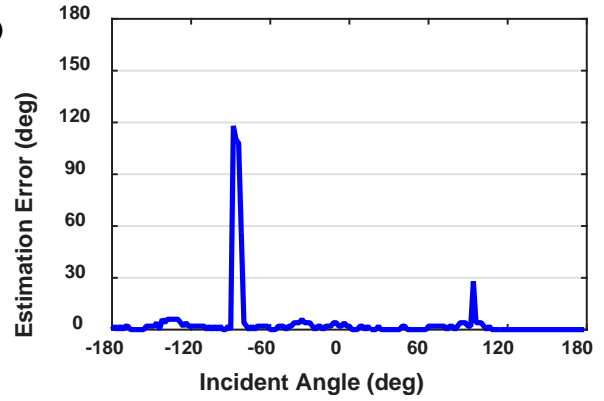

(c)

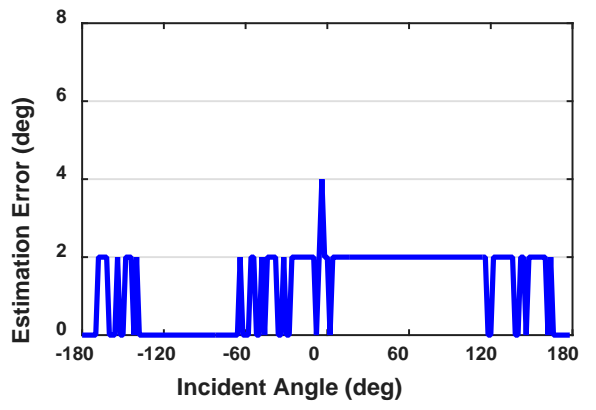

Fig. 14. The estimation errors of the D-shaped UWB antenna with incident waves in (a) the $x-y$ plane (H-field in the $z$ direction), (b) the $x$-z plane (H-field in the $y$ direction), and (c) the $y$-z plane (E-field in the $x$ direction).

\section{CONCLUSION AND DISCUSSION}

In this paper, a DoA estimation technique using a single UWB antenna inspired by the human auditory system is investigated. Because the received spectra of the UWB antenna are incident angle dependent, the DoA of a broadband signal can be estimated from the correlation coefficients of the measured spectra and pre-determined calibration spectra. Both an elliptical UWB antenna and an unsymmetrical D-shaped UWB antenna are studied for the DoA estimation applications. The feasibility of the single antenna DoA estimation technique is demonstrated in both simulations and experiments.

Compared to previous work using two antennas and MUSIC algorithm [6, 7] which achieved $1^{\circ}$ to $2^{\circ}$ accuracy, this single antenna approach has the advantages of requiring less hardware components and less computational intensity (no Eigenvalue calculations needed) at the expense of sacrificing some accuracy. This kind of biological inspired RF technique may lead to future novel direction finding systems that are low-cost, compact and lightweight.

Although with its advantages, before practical applications could be widely implemented, several potential issues of this technique need to be addressed. First, our initial design (the elliptical antenna) indicates that good SNR is needed to obtain high DoA estimation accuracy using this technique due to some intrinsic symmetries of the antenna. Nevertheless, with an improved antenna design (D-shaped to break some of the symmetries), the DoA estimation performance is better compared to the elliptical UWB antenna under the same SNR conditions. It is likely that further improvement of the antenna design with higher incident angle dependent spectra can be achieved for better performance. In addition, although the UWB band (3.1 to $10.6 \mathrm{GHz}$ ) is used to demonstrate the idea here, the technique itself should work at other frequency bands as well so that higher SNR scenarios may be realized. Furthermore, as analyzed, high transmitted power spectral distortion and strong multipath effect will degrade the DoA estimation performance. Finally, in our experiments, the cable scattering may affect the resulting spectra, while in practical applications the placement of the feeding line should be carefully taken into consideration to eliminate this issue.

\section{ACKNOWLEDGEMENT}

This work was supported in part by the U.S. Army Research Laboratory and the U.S. Army Research Office under agreement number W911NF-1-01-0285.

\section{REFERENCES}

[1] S. E. Lipsky, Microwave Passive Direction Finding, John Wiley \& Sons, Inc., 1987.

[2] L.C. Godara, "Application of antenna arrays to mobile communications. II. Beam-forming and direction-of-arrival considerations," Proc. IEEE, vol. 85, no. 8, pp. 1195-1245, 1997.

[3] R. Roy and T. Kailath, "ESPRIT-estimation of signal parameters via rotational invariance techniques," IEEE Trans. Acoust., Speech, Signal Processing, vol. 37, no. 7, pp. 984-995, July 1989.

[4] R. O. Schmid, "Multiple emitter location and signal parameter estimation,” IEEE Trans. Antennas Propagat., vol. 34, pp. 276-280, 1986.

[5] H. Xin and J. Ding, "An improved two-antenna direction of arrival (DoA) technique inspired by human ears,” IEEE AP-S Intl Symp. Dig., pp. 1-4, July. 2008.

[6] R. Zhou, H. Zhang and H. Xin, "Improved Two-Antenna Direction Finding Inspired by Human Ears," IEEE Trans. Antennas Propagat., Vol. 59, pp. 2691-2697, July 2011.

[7] N. Behdad, M. A. Al-Joumayly and M. Li, "Biologically Inspired Electrically Small Antenna Arrays with Enhanced Directional Sensitivity," IEEE Ant. And Wireless Prop. Letts., vol. 10, pp. 361-364, 2011.

[8] R. A. Butler, "The monaural localization of tonal stimuli," Percept. Psychophys., vol. 9, pp. 99-101, 1971.

[9] J. Blauert, "Sound localization in median plane," Acoustica, vol. 22, pp. 206-213, 1969.

[10] M. M. Van Wanrooij, Monaural adaptive mechanisms in human sound localization, Ph.D. dissertation, Dept. Biophysics, Radboud Univ., Nijmegen, Netherlands, 2007.

[11] J. Oppermann, M. Hamalainen, and J. Linatti, UWB Theory and Applications, John Wiley \& Sons, Inc., 2004.

[12] R. Zhou and H. Xin, "A novel direction of arrival estimation technique using a single UWB antenna,” IEEE APSURSI Intl Symp., July, 2010

[13] H. Zhang, R. Zhou, Z. Wu, H. Xin, and R. W. Ziolkowski, "Designs of ultra wideband (UWB) printed elliptical monopole antennas with slots," Microw. Opt. Technol. Lett., vol. 52, pp. 466-471, 2010.

[14] Ansoft High Frequency Structure Simulator (HFSS) V. 11.1.

[15] M. K. Samimi, and T. S. Rappaport, "Statistical Channel Model with Multi-Frequency and Arbitrary Antenna Beamwidth for Millimeter-Wave Outdoor Communications," IEEE Global Telecommunications Conference (GLOBECOM), Dec., 2015.

[16] X. Yu and H. Xin, "Direction of Arrival Estimation Utilizing Incident Angle Dependent Spectra,” IEEE MTT-S Intl Symp., June, 2012. 